\title{
Remarks on the Harnak Inequality for Local-Minima of Scalar Integral Functionals with General Growth Conditions
}

\author{
Tiziano Granucci \\ Scuola Superiore, Istituto P. Calamandrei, Firenze, Italy \\ Email: tizianogranucci@libero.it
}

Received September 2013

\section{Abstract \\ In this paper we proof a Harnack inequality and a regularity theorem for local-minima of scalar intagral functionals with general growth conditions.}

\section{Keywords}

\section{Harnack Inequality, Regularity, Hölder Continuity}

\section{Introduction}

In this paper we proof a Harnack inequality for local-minima of scalar intagral functionals of the calculus of variation of that type

$$
\mathrm{J}[u, \Omega]=\int_{\Omega} f(x, u(x), \nabla u(x)) d x
$$

where $\Omega$ is a bounded open subset of $\mathbb{R}^{N}, \Phi:[0,+\infty) \rightarrow[0,+\infty)$ is a $\mathrm{N}$-function and $\Phi$ globally satisfies the $\Delta^{\prime}$ condition in $[0,+\infty)$, f : $\Omega \times \mathbb{R} \times \mathbb{R}^{\mathrm{N}} \rightarrow \mathbb{R}$ is a Carathéodory function and there exist $\mathrm{L}_{1}, \mathrm{~L}_{2} \in(0,+\infty) \mathrm{L}_{2}$ and

$$
\Phi(|z|) \leq f(x, s, z) \leq L_{2} \Phi(|z|)
$$

for a. e. $x \in \Omega$ and for every $(s, z) \in \Omega^{\prime} \times \Omega^{N}{ }^{N}$. The risearch of regularity results for elliptic and parabolic equations start from the basic and most important results of E. De Giorgi [5] and J. Nash [27]. In 1990s, beginning from the papers of G. Astarita and G. Marrucci [3] and J. P. Gosez [13] has been developed a remarkable production of regularity results for functionals with general growths. In [7], [8] and [25], M. Fuchs, G. Mingione, G. Seregin and F. Siepe have studied functionals of the type

$$
J[u, \Omega]=\int \Omega \nabla u(x) \ln (1+|\nabla u(x)|) d x
$$

showing results of partial and global regularity for the minimizer of such functional in the scalar and vectorial case. Moreover in [8] M. Fuchs and G. Mingione, have already studied functionals of this type

$$
J[u, \Omega]=\int_{\Omega} \Phi(|\nabla u|) d x .
$$


In papers $[7,8,25]$ the regularity of the minimizer of the functionals (1.2) and (1.3) has been obtained starting from the weak Eulero-Lagrange equations using the hypothesis: $\Phi \in \mathrm{C}^{2}$. We remember that in $[7,8,25]$ there are important estimations on the $L^{\wedge}\{\infty\}$ norm of the gradient of the minima both in the scalar case and in the vectorial one. In [24] E. Mascolo and G. Papi have determined an inequality of Harnack for the minimizer of the functional (1.3) under the condition $\Phi \in \Delta_{2} \cap \nabla_{2}$. We observe that $\Phi \in \Delta_{2} \cap \nabla_{2}$ implies

$$
t^{p}-c_{2}<\Phi(t)<c_{3} t^{m}+c_{4} \text { for } t>0
$$

with real positive constants $\mathrm{c}_{1}, \mathrm{c}_{2}, \mathrm{c}_{3}, \mathrm{c}_{4}$ and $1<\mathrm{p} \leq \mathrm{m}$. Therefore the functional (1.3) satisfies non-standard growth conditions. Classical regularity theorem for functionals with standard growth conditions $(p=m)$ has been proved in [9] and [10] (for a didactic explanation refer to [2,11,12]). In [26], G. Moscariello and L. Nania has obtain a results of hölder continuity for the local-minima of functional of the type (1.1) under the hypothesis that (1.4) holds with $1<\mathrm{p} \leq \mathrm{m}<((\mathrm{Np}) /(\mathrm{N}-\mathrm{p}))$. In [17], G. M. Lieberman proved an Harnack inequality for the local-minima of the functional (1.1) with $\Phi \in \mathrm{C}^{2}$ suth that verifies the following relation

$$
c_{5} \leq t \Phi^{\prime \prime}(t) / \Phi^{\prime}(t) \leq c_{6} \text { for } t>0
$$

with $0<\mathrm{c}_{5}<\mathrm{c}_{6}$. We are interested in functionals with quasi-linear growths and we will proof a regularity result which extend the ones obtained in $[17,24,26]$ to a wider N-functional class. In particular we get that the localminima of the following functionals:

$$
J[u, \Omega]=\int_{\Omega}|\nabla u|^{p} \ln (1+|\nabla u|) d x \text { with } p>1
$$

are holder continuous functions. In [14] and [15] we start to study the regularity of the local-minima introducing a maximal $L^{\Phi}-L^{\infty}$ inequality and estimating the measure of the level set $A(k, R)$. Moreover in [15] and [16] we have shown that the following hypothesis can be used in order to give a new estimation of the measure of the livel set $A(k, R)$ :

$H-1) \Phi$ globally satisfies the $\Delta^{\prime}$-condition in $[0,+\infty)$;

$\mathrm{H}-2)$ there exists a constant $\mathrm{c}_{\mathrm{H}_{2}}>0$

$$
\Phi(t) \Phi(1 / t) \leq c_{H_{2}} \text { for every } t \in(0,1)
$$

H-3) there exists a constant $c_{\mathrm{H}_{3}}>0$

$$
\Phi^{-1}(t) \leq c_{H_{3}} t^{1 / m} \text { for every } t \in(0,1) .
$$

Under these hypotheses we can show the following result.

Theorem 1: If $\mathrm{u} \in \mathrm{W}^{1} \mathrm{~L}^{\Phi}(\Omega)$ is a quasi-minima of the functional (1.1) and if $\Phi$ confirm the hypotheses H-1, $\mathrm{H}-2$ and $\mathrm{H}-3$; then $\mathrm{u}$ is locally hölder continuous.

In these pages we show that the hypotheses $\mathrm{H}-2$ and $\mathrm{H}-3$ are purely technical and they can be eliminated. We can subsequently weaken besides H-1.

We will suppose that the following hypothesis hold.

G-1) Let $\varpi: \mathbb{R}^{+} \rightarrow \mathbb{R}^{+}$be an increasing function such that

$$
\Phi(\varepsilon t) \leq c_{G} \varepsilon \varpi(\varepsilon) \Phi(t)
$$

for every $\mathrm{t} \in \mathbb{R}^{+}$and for every $\varepsilon \in(0,1)$, where $\mathrm{c}_{\mathrm{G}}>0$ is a real constant. Moreover we suppose that

$$
\lim _{x \rightarrow 0^{+}} \varpi(s)=0 .
$$

We say that $\Phi \in \mathrm{G}$ if (1.8) holds. The hypothesis G-1 implicates a type of quasi-sub-homogeneity condition on the $\mathrm{N}$-function $\Phi$.

Remark 1: We observe that if $\Phi \in \Delta_{2} \cap \nabla_{2}$ then by Lemma 3 (i) we have

$$
\Phi(\varepsilon t)=\varepsilon^{r}\left(1 / \varepsilon^{r}\right) \Phi(\varepsilon t) \leq \varepsilon^{r} \Phi(t) .
$$

Then the functions $\Phi \in \Delta_{2} \cap \nabla_{2}$ verify the hypothesis G-1.

Remark 2: We observe that if $\Phi \in \Delta^{\prime}$ on $(0,+\infty)$ then $\Phi$ verify the hypothesis G-1; in fact

$$
\Phi(\varepsilon t) \leq c \Phi(\varepsilon) \Phi(t) .
$$


Our principal results will be, a weak inequality of Harnack [Theorem 5] and the corollary of regularity that it follows of it [Corollary 2]. The proof of the Harnack inequality uses the techniques introduced in $[6,17]$ and $[24]$. The only present novelty in the demonstrative technique is the use of an $\varepsilon$-Young inequality. This simple trick allows to recover the results introduced in $[15-17,24,26]$ in a simple way and without using the properties of the functions $\Delta_{2} \cap \nabla_{2}$ (see Lemma of [15,24] and [26]). We finally observe that the hypotheses $\Delta_{2} \cap \nabla_{2}$ it is not, in general, equivalent to H-1; therefore the hypothesis G-1 seems to be slightly more general of those introduced in [15-17,24,26].

Definition 1: Let $\mathrm{p}$ be a real valued function defined on $[0,+\infty)$ and having the following properties: $\mathrm{p}(0)=0$, $p(t)>0$ if $t>0, p$ is nondecreasing and right continuous on $(0,+\infty)$. Then the real valued function $\Phi$ defined on $[0,+\infty)$ by

$$
\Phi(t)=\int_{[0, t]} p(s) d s
$$

is called an $\mathrm{N}$-function.

The function $\Phi:[0,+\infty) \rightarrow[0,+\infty)$ defined by (1.9) satisfies the following properties:

$$
\begin{gathered}
\Phi(0)=0 \text { and } \Phi(t)>0 \text { if } t>0 ; \\
\Phi \text { is continuous on }[0,+\infty) ; \\
\Phi \text { is strictly increasing on }[0,+\infty) ; \\
\Phi \text { is convex on }[0,+\infty) ; \\
\lim _{x \rightarrow 0} \Phi(t) / t=0 \text { and } \lim _{x \rightarrow \infty} \Phi(t) / t=+\infty \text {, } \\
\text { if } s>t>0 \text {, then } \Phi(s) / s>\Phi(t) / t .
\end{gathered}
$$

Definition 2: Let $\mathrm{p}$ be a real valued function defined on $[0,+\infty)$ and having the following properties: $\mathrm{p}(0)=0$, $p(t)>0$ if $t>0, p$ is nondecreasing and right continuous on $(0,+\infty)$. We define

$$
q(s)=\sup _{p(t) \leq s}(t)
$$

and

$$
\Psi(t)=\int_{[0, t]} q(s) d s .
$$

The N-functions $\Phi$ and $\Psi$ given by (1.9) and (1.10) are said to be complementary.

Particularly for us it will be important the following Lemma.

Lemma 1: Let $\Phi$ be an $\mathrm{N}$-function, let $\Psi$ be the complemantary $\mathrm{N}$-function of $\Phi$ then we have

$$
s t \leq \Phi(s)+\Psi(t)
$$

$\forall \mathrm{s}, \mathrm{t} \in \mathbb{R}^{+}$. Moreover for every $\varepsilon>0$ we get

$$
s t \leq(1 / \varepsilon) \Phi(\varepsilon s)+(1 / \varepsilon) \Psi(t) \forall s, t \in \mathbf{i}^{+} .
$$

Definition 3: A N-function $\Phi$ is of class $\Delta_{2}$ globally in $(0,+\infty)$ if exists $\mathrm{k}>1$ such that

$$
\Phi(2 t) \leq k \Phi(t) \forall t \in(0,+\infty) .
$$

Definition 4: A N-function $\Phi$ is of class $\Delta_{2} \wedge\{\mathrm{m}\}$ globally in $(0,+\infty)$, with $\mathrm{m}>1$, if for every $\lambda>1$

$$
\Phi(\lambda t) \leq \lambda^{m} \Phi(t) \forall t \in(0,+\infty) .
$$

The N-functions $\Phi \in \Delta_{2}{ }^{\mathrm{m}}$ are characterized by the following result

Lemma 2: Let $\Phi$ be a $\mathrm{N}$-function and let $\Phi_{-}^{\prime}$ be its left derivative. For $\mathrm{m}>1$ the following properties are equivalent:

1) $\Phi(\lambda t) \leq \lambda^{m} \Phi(t)$, for every $t \geq 0$, for every $\lambda>1$;

2) $t \Phi_{-}^{\prime}(t) \leq m \Phi(t)$, for every $t \geq 0$;

3) the function $\Phi(t) / t^{r}$ is non-increasing on $(0,+\infty)$. 
The $\mathrm{N}$-functions $\Phi \in \nabla_{2}{ }^{\mathrm{r}}$ are characterized by the following result

Lemma 3: Let $\Phi$ be a $\mathrm{N}$-function and let $\Phi^{\prime}$ - be its left derivative. For $r>1$ the following properties are equivalent:

1) $\Phi(\lambda t) \geq \lambda^{\mathrm{r}} \Phi(\mathrm{t})$, for every $\mathrm{t} \geq 0$, for every $\lambda>1$;

2) $t \Phi^{\prime}-(t) \geq r \Phi(t)$, for every $t \geq 0$;

3) the function $\Phi(\mathrm{t}) / \lambda^{\mathrm{r}}$ is non-decreasing on $(0,+\infty)$.

Definition 5: We say that a N-function $\Phi$ belongs to the class $\Phi \in \nabla_{2}{ }^{r}$ if any of the three condition (i)', (ii)' or (iii)' is satisfied.

Definition 6: We say that the $\mathrm{N}$-function $\Phi$ satisfies the $\Delta^{\prime}$-condition if there exist positive constants-c and $\mathrm{t}_{0}$ - such that

for every $\mathrm{t}, \mathrm{s} \geq \mathrm{t}_{0}$.

$$
\Phi(t s) \leq c_{4} \Phi(t) \Phi(s)
$$

Definition 7: We say that the $\mathrm{N}$-function $\Phi$ globally satisfies the $\Delta^{\prime}$-condition in $[0,+\infty)$ if $(1.12)$ holds for every $\mathrm{t}, \mathrm{s} \geq 0$.

We remember that if $\Phi \in \mathrm{C}^{2}$ then $\Phi \in \Delta^{\prime}$ if $\mathrm{t} \Phi^{\prime \prime}(\mathrm{t}) / \Phi^{\prime}(\mathrm{t})$ is a non-increasing function, for further details refer to Theorems 5.1 and 5.2 and to the Lemma 5.2 of [19].

Lemma 4: If the $\mathrm{N}$-function $\Phi$ satisfies the $\Delta^{\prime}$-condition then it also satisfies the $\Delta_{2}$-condition

The $\mathrm{N}$-functions

$$
\begin{gathered}
\Phi_{1}(t)=t^{p} \text { with } p>1 ; \\
\Phi_{2}(t)=t^{p}(\ln (t) \mid+1) \text { with } p>1 ; \\
\Phi_{3}(t)=(1+t) \ln (1+t)-t ; \\
\Phi_{4}(t)=\left(t^{2}\right) /(1+\ln (1+t)) .
\end{gathered}
$$

satisfy the $\Delta^{\prime}$-condition. Moreover $\Phi_{1}$ and $\Phi_{2}$ satisfy the $\Delta^{\prime}$-condition globally in $[0,+\infty)$ and belong to the class $\nabla_{2}$ globally in $[0,+\infty)$. The function $\Phi_{3}$ does not satisfy $\Delta^{\prime}$-condition for all t,s $\geq 0$ and $\Phi_{3} \notin \nabla_{2}$. Osseviamo inoltre che la funzione $\Phi_{4} \in \nabla_{2} \cap \Delta_{2}$ but $\Phi_{4}$ does not satisfy the $\Delta^{\prime}$-condition. For further details refer to $[1,19,28]$. Now we can introduce Orlicz spaces and Orlicz Sobolev Spaces, $\mathrm{L}^{\Phi}$ and $\mathrm{W}^{1} \mathrm{~L}^{\Phi}$; in these definitions and throughout the article we assume that $\Phi$ is a $\mathrm{N}$-function of class $\Delta_{2}{ }^{\mathrm{m}}$ for some $\mathrm{m}>1$ and that $\Omega \subset \mathbb{R}^{\mathrm{N}}$ is a bounded open set with Lipschitz boundary.

Definition 8: If $\mathrm{u}$ is a $\mathrm{L}^{\mathrm{N}}$-measurable function on $\Omega$ and: $\int_{\Omega} \Phi(|\mathrm{u}|) \mathrm{dx}<+\infty$ then $\mathrm{u} \in \mathrm{L}^{\Phi}(\Omega)$. Moreover

$$
W^{1} L^{\Phi}(\Omega)=\left\{u \in L^{\Phi}(\Omega): \partial_{i} u \in L^{\Phi}(\Omega) \text { for } i=1, \ldots, N\right\}
$$

where $\partial_{\mathrm{i}} \mathrm{u}$, for $\mathrm{I}=1, \ldots, \mathrm{N}$, are the weak derivatives of $\mathrm{u}$.

Theorem 2: $\mathrm{L}^{\Phi}(\Omega)$ e $\mathrm{W}^{1} \mathrm{~L}^{\Phi}(\Omega)$ are Banach spaces with the following norms

$$
\|u\|_{\Phi, \Omega}=\inf \left(k>0: \int_{\Omega} \Phi((|u|) / k) d x \leq 1\right)
$$

and

$$
\|u\|_{1, \Phi, \Omega}=\|u\|_{\Phi, \Omega}+\sum_{i=1, \ldots, N}\left\|\partial_{i} u\right\|_{\Phi, \Omega}
$$

For greater details we refer to $[1,19,28]$. If $\mathrm{u} \in \mathrm{W}_{\text {loc }}{ }^{1} \mathrm{~L}^{\Phi}(\Omega), \mathrm{k}$ is a real number and $\mathrm{Q}_{\mathrm{R}} \Subset \Omega$, we set

$$
\begin{aligned}
& A(k, R)=\left\{x \in Q_{R}: u(x)>k\right\}=\{u>k\} \cap Q_{R}, \\
& B(k, R)=\left\{x \in Q_{R}: u(x)<k\right\}=\{u<k\} \cap Q_{R} .
\end{aligned}
$$

Remark 3: For almost each $\mathrm{k} \in \mathbb{R}$ we get $|\mathrm{A}(\mathrm{k}, \mathrm{R})|=\left|\mathrm{Q}_{\mathrm{R}}\right|-|\mathrm{B}(\mathrm{k}, \mathrm{R})|$.

Definition 9: If $\mathrm{u} \in \mathrm{W}_{\text {loc }}{ }^{1} \mathrm{~L}^{\Phi}(\Omega)$, we say that $\mathrm{u} \in \mathrm{ODG}_{\Phi}^{+}(\Omega, \mathrm{H}, \mathrm{R})$ if for every couple of concentric balls $\mathrm{Q}_{\varrho} \subset \mathrm{Q}_{\mathrm{R}} \Subset \mathrm{Q}_{\mathrm{R}_{0}} \Subset \Omega$, with $\mathrm{R}<\mathrm{R}_{0}$, and for every $\mathrm{k} \in \mathbb{R}$ we have

$$
\int_{A(k, \tilde{\mathbf{n}})} \Phi(|\nabla u|) d x \leq H \int_{A(k, R)} \Phi((u-k) /(R-\widetilde{\mathbf{n}})) d x
$$


Definition 10: If $\mathrm{u} \in \mathrm{W}_{\mathrm{loc}}{ }^{1} \mathrm{~L}^{\Phi}(\Omega)$, we say that $\mathrm{u} \in \mathrm{ODG}_{\Phi}{ }^{-}\left(\Omega, \mathrm{H}, \mathrm{R}_{0}\right)$ if for every couple of concentric balls $\mathrm{Q}_{\varrho} \subset \mathrm{Q}_{\mathrm{R}} \Subset \mathrm{Q}_{\mathrm{R}_{0}} \Subset \Omega$, with $\mathrm{R}<\mathrm{R}_{0}$, and for every $\mathrm{k} \in \mathbb{R}$ we have

$$
\int_{B(k, \tilde{\mathbf{n}})} \Phi(|\nabla u|) d x \leq H \int_{B(k, R)} \Phi((k-u) /(R-\widetilde{\mathbf{n}})) d x
$$

Definition 11: If $\mathrm{u} \in \mathrm{W}_{\text {loc }}{ }^{1} \mathrm{~L}^{\Phi}(\Omega)$, we say that $\mathrm{u} \in \mathrm{ODG}_{-}\{\Phi\}\left(\Omega, \mathrm{H}, \mathrm{R}_{0}\right)$ if $\mathrm{u} \in \mathrm{ODG}_{-}\{\Phi\}^{\wedge}\{ \pm\}\left(\Omega, \mathrm{H}, \mathrm{R}_{0}\right)$, that is

$$
O D G_{\Phi}\left(\Omega, H, R_{0}\right)=O D G_{\Phi}{ }^{+}\left(\Omega, H, R_{0}\right) \cap O D G_{\Phi}{ }^{-}\left(\Omega, H, R_{0}\right) .
$$

Theorem 3: If $\mathrm{u} \in \mathrm{ODG}_{\Phi}{ }^{+}\left(\Omega, \mathrm{H}, \mathrm{R}_{0}\right)$ then $\mathrm{u}$ is locally bounded above on $\Omega$. Furthermore, for each $\mathrm{x}_{0} \in \Omega$ and $\mathrm{R}$ $\leq \min \left(\mathrm{R}_{0}, \mathrm{~d}\left(\mathrm{x}_{0}, \partial \Omega\right), 1\right)$ there exists an universal constant $\mathrm{c}_{5}=\mathrm{c}_{5}(\mathrm{~N}, \mathrm{~m}, \mathrm{H})$ such that

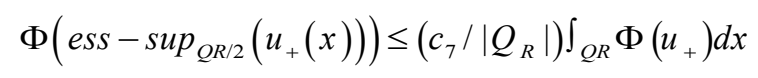

Proof: The proof follows using the demonstration methods presented in [24].

Corollary 1: If $\mathrm{u} \in \mathrm{ODG}_{\Phi}\left(\Omega, \mathrm{H}, \mathrm{R}_{0}\right)$ then $\mathrm{u}$ is locally bounded on $\Omega$. Furthermore, for each $\mathrm{x}_{0} \in \Omega$ and $\mathrm{R} \leq$ $\min \left(\mathrm{R}_{0}, \mathrm{~d}\left(\mathrm{x}_{0}, \partial \Omega\right), 1\right)$ there exists an universal constant $\mathrm{c}_{6}=\mathrm{c}_{6}(\mathrm{~N}, \mathrm{~m}, \mathrm{H})$ such that

$$
\Phi\left(\operatorname{ess}-\sup _{Q R / 2}(|u(x)|)\right) \leq\left(c_{7} /\left|Q_{R}\right|\right) \int_{Q R} \Phi(|u|) d x .
$$

Proof: The proof comes after Theorem 3 remembering that if $u \in \mathrm{DG}_{\Phi}{ }^{-}\left(\Omega, H, \mathrm{R}_{0}\right)$ then $-\mathrm{u} \in \mathrm{DG}_{\Phi}{ }^{+}\left(\Omega, H, \mathrm{R}_{0}\right)$.

Moreover the following lemma is valid:

Lemma 5: If $\mathrm{u} \in \mathrm{DG}_{\Phi}{ }^{+}\left(\Omega, \mathrm{H}, \mathrm{R}_{0}\right)$ then $\mathrm{u}$ is locally bounded above on $\Omega$. Furthermore, for each $\mathrm{x}_{0} \in \Omega, \mathrm{R} \leq$ $\min \left(\mathrm{R}_{0}, \mathrm{~d}\left(\mathrm{x}_{0}, \partial \Omega\right), 1\right)$ and for every $\mathrm{p}>1$ there exists an universal constant $\mathrm{c}_{7}=\mathrm{c}_{7}(\mathrm{p}, \mathrm{N}, \mathrm{m}, \mathrm{H})$ such that

$$
\left.\Phi^{1 / p}\left(\operatorname{ess}-\sup _{Q \widetilde{\mathbf{n}}}|u|\right)\right) \leq\left(c_{7} /(R-\widetilde{\mathbf{n}})^{N}\right) \int_{Q R} \Phi^{1 / p}(|u|) d x
$$

for each $\mathrm{Q}_{\varrho} \Subset \mathrm{Q}_{\mathrm{R}}$ and $0<\varrho<\mathrm{R}$.

Proof: The proof comes after Theorem 3 using the demonstration methods presented in [24].

Definition 12: Let $\mathrm{u} \in \mathrm{W}_{\text {loc }}{ }^{1} \mathrm{~L}^{\Phi}(\Omega)$ then it is a local minima of $(1.1)$ if for every $\phi \in \mathrm{W}_{0}{ }^{1} \mathrm{~L}^{\Phi}(\Omega)$ we have

$$
J(u, \operatorname{supp}(\phi)) \leq J(u+\phi, \operatorname{supp}(\phi)) .
$$

Moreover we get:

Theorem 4 (Caccioppoli inequalities): If $\Phi \in \Delta_{2}$ and $\mathrm{u} \in \mathrm{W}_{\text {loc }}{ }^{1} \mathrm{~L}^{\Phi}(\Omega)$ is a local minima of (1.1) then $\mathrm{u} \in \mathrm{ODG}_{\Phi}\left(\Omega, \mathrm{H}, \mathrm{R}_{0}\right)$.

Using the previous results we obtain the following theorems:

Theorem 5 (Weak Harnack inequality): Let $\Phi$ be a $\mathrm{N}$-function. Let $\mathrm{u}$ be a positive function satisfying $(1,17)$. If $\Phi \in \mathrm{G}$; then there exists $\mathrm{p}>1$ and a constant $\mathrm{c}>0$ such that

$$
\Phi^{1 / p}\left(e s s-i n f_{Q R / 2}(u)\right) \geq c\left(1 / R^{N}\right) \int_{Q R} \Phi^{1 / p}(u) d x .
$$

Theorem 6 (Main Theorem-Harnack inequality): Let $\Phi$ be a N-function. Let $\mathrm{u}$ be a positive local minimizer of (1.1). If $\Phi \in \mathrm{G}$; then there exists a constant $\mathrm{c}>0$ such that, for $\sigma \in(0,1)$ we have

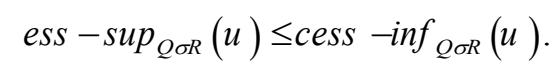

Proof (Proof of the Main Theorem): Using the (1.21) and (1.22) we have (1.23).

Corollary 2: Let $\Phi$ be a $\mathrm{N}$-function. If $\Phi \in \mathrm{G}$ and $\mathrm{u} \in \mathrm{W}^{1} \mathrm{~L}^{\Phi}(\Omega)$ is a local minimizer of the functional (1.1); then $\mathrm{u}$ is locally hölder continuous.

Proof: Using (1.20) and the technique introduced in $[6,11,12]$ we get the proof.

We finish observing that with small changes our demonstrative technique can also be applied to the quasi-minima of the functional (1.1). Besides we can also apply this demonstration using equivalent $\mathrm{N}$-functions. Unfortunately, $\Phi_{2}(\mathrm{t})=\operatorname{tln}(1+\mathrm{t})$ does not verify H1; for this $\Phi_{2} \in \Delta^{\prime}$ on $\left[\mathrm{t}_{0},+\infty\right)$ with $\mathrm{t}_{0}>0$ but $\Phi_{2} \notin \Delta^{\prime}$ globally on $[0,+\infty)$. We should think to solve this problem using the concept of equivalent $\mathrm{N}$-function; the demonstrative technique allows it, but we do not know if it exists a $\mathrm{N}$-function $\Phi_{3}$ equivalent to $\Phi_{2}$ which globally verifies $\Delta^{\prime}$ globally on $[0,+\infty)$. It is still an unsolved problem.I thank the colleague Dott. Elisa Albano who translated the article into English supporting and encouraging me so much. 


\section{Proof of the Weak Harnack Inequality}

\subsection{Lemmata}

Let define

$$
v_{R}(y)=((u(R y)) / R), y \in Q_{1}
$$

then we have the following Caccioppoli inequalities

$$
\int_{A(k, \sigma, v R)} \Phi\left(\left|\nabla v_{R}\right|\right) d x \leq H \int_{A(k, \tau, v R)} \Phi\left(\left(\left(v_{R}-k\right) /(\tau-\sigma)\right)\right) d x
$$

and

$$
\int_{B(k, \sigma, v R)} \Phi\left(\left|\nabla v_{R}\right|\right) d x \leq H \int_{B(k, \tau, \nu R)} \Phi\left(\left(\left(k-v_{R}\right) /(\tau-\sigma)\right)\right) d x
$$

where $0<\sigma<\tau<1$ and $\mathrm{k} \in \mathbb{R}$.

Let us start remembering the following lemma:

Lemma 6: Let $\mathrm{g}(\mathrm{t}), \mathrm{h}(\mathrm{t})$ be a non-negative and increasing functions on $[0,+\infty)$ then $\mathrm{g}(\mathrm{t}) \mathrm{h}(\mathrm{s}) \leq \mathrm{g}(\mathrm{t}) \mathrm{h}(\mathrm{t})+\mathrm{g}(\mathrm{s}) \mathrm{h}(\mathrm{s})$ for every $\mathrm{s}, \mathrm{t} \in[0,+\infty)$.

Proof: If $\mathrm{s} \leq \mathrm{t}$ then $\mathrm{g}(\mathrm{t}) \mathrm{h}(\mathrm{s}) \leq \mathrm{g}(\mathrm{t}) \mathrm{h}(\mathrm{t}) \leq \mathrm{g}(\mathrm{t}) \mathrm{h}(\mathrm{t})+\mathrm{g}(\mathrm{s}) \mathrm{h}(\mathrm{s})$. If $\mathrm{t} \leq \mathrm{s}$ then $\mathrm{g}(\mathrm{t}) \mathrm{h}(\mathrm{s}) \leq \mathrm{g}(\mathrm{s}) \mathrm{h}(\mathrm{s}) \leq \mathrm{g}(\mathrm{t}) \mathrm{h}(\mathrm{t})+\mathrm{g}(\mathrm{s}) \mathrm{h}(\mathrm{s})$.

Let us remember for the sake of completeness the following lemma:

Lemma 7: Let $\Phi \in \Delta_{2}$ and $\mathrm{u} \in \mathrm{W}^{1} \mathrm{~L}^{\Phi}(\Omega)$. Suppose that $\mathrm{u}$ is positive in $\mathrm{Q}_{\mathrm{R}}$ and satisfies (2.2) then there exists a positive constants $\delta_{0}$ such that if for some $\theta>0$ we have $|\mathrm{B}(\theta, \mathrm{u}, \mathrm{R})| \leq \delta_{0}\left|\mathrm{Q}_{\mathrm{R}}\right|$, then

$$
\inf _{Q R / 2}\{u\} \geq(\theta / 2) \text {. }
$$

Proof: The proof follows using the demonstration methods presented in [24]. Refer to Lemma 4.1 of [24].

Our demonstration of the weak inequality of Harnack founds him on the following Lemma 8 . We have shown the Lemma 8 using an opportune $\varepsilon$-Young inequality.

Lemma 8: Let be $\Phi$ a $\mathrm{N}$-function and $\Phi \in \mathrm{G}$. Let $\mathrm{u} \in \mathrm{W}^{1} \mathrm{~L}^{\Phi}(\Omega)$. Suppose that $\mathrm{u}$ satisfies (2.2). For every $\delta \in(0,1)$ and $\mathrm{T}>(1 / 2)$, there exists a positive constant $\mu(\delta, T)$ such that if $\mathrm{u}$ is positive on $\mathrm{Q}_{2 \mathrm{TR}}$ and there exists $\theta>$ 0 such that $|\mathrm{B}(\theta, \mathrm{u}, \mathrm{R})| \leq \delta\left|\mathrm{Q}_{\mathrm{R}}\right|$, we have

$$
\inf _{\text {QTR }}\{u\} \geq \mu(\delta, T) \theta \text {. }
$$

Proof: Let $\delta \in(0,1)$. We first prove that if $\mathrm{u}$ is positive in $\mathrm{Q}_{\mathrm{R}}$ and there exists $\theta>0$ such that $|\mathrm{B}(\theta, \mathrm{u}, \mathrm{R})|<\delta\left|\mathrm{Q}_{\mathrm{R}}\right|$, there exists a constant $\lambda(\theta)$ such that

$$
\inf _{Q R / 2}\{u\} \geq \lambda(\theta) \theta
$$

We consider the function $w_{R}$ define by $w_{R}(y)=0$ if $v_{R}(y) \geq k, w_{R}(y)=k-v_{R}$ if $k>v_{R}(y)>h, w_{R}(y)=k-h$ if $\mathrm{v}_{\mathrm{R}}(\mathrm{y}) \leq \mathrm{h}$

where $v_{R}(y)=((u(R y)) / R), y \in Q_{1}$. Let us consider $k_{i}=\left(\theta /\left(2^{i} R\right)\right)$ with $I \leq v$, since $w_{R}=0$ in $Q_{1} \backslash B\left(k_{i}, v_{R}, 1\right)$ and

$$
\left|Q_{1} \backslash B\left(k_{i}, v_{R}, 1\right)\right|>(1-\delta)\left|Q_{R}\right|
$$

by Sobolev inequality we have

$$
\Phi\left(k_{i}-k_{i-1}\right)\left|B\left(k_{i}, v_{R}, 1\right)\right| \leq\left|B\left(k_{i}, v_{R}, 1\right)\right|^{1 / N}\left[\int_{Q_{1}}\left(\Phi\left(w_{R}\right)\right)^{N /(N-1)} d x\right]^{(N-1) / N)}
$$

and

$$
\Phi\left(k_{i}-k_{i-1}\right)\left|B\left(k_{i}, v_{R}, 1\right)\right| \leq\left. C_{S N}|| B\left(k_{i}, v_{R}, 1\right)\right|^{1 / N} \int_{\Delta} \Phi^{\prime}\left(w_{R}\right)\left|\nabla w_{R}\right| d x
$$

where $\Delta_{\mathrm{i}}=\mathrm{B}\left(\mathrm{k}_{\mathrm{i}}, \mathrm{V}_{\mathrm{R}}, 1\right) \backslash \mathrm{B}\left(\mathrm{k}_{\mathrm{i}-1}, \mathrm{~V}_{\mathrm{R}}, 1\right)$. Using the Young inequality ab $\leq \Psi(\mathrm{a})+\Phi(\mathrm{b})$, where $\Phi$ is the complementary function of $\Phi$, we have

$$
\int_{\Delta i} \Phi\left(w_{R}\right)\left|\nabla w_{R}\right| d x=(m / \varepsilon) \int_{\Delta i}\left(\Phi^{\prime}\left(w_{R} / m\right) \varepsilon\left|\nabla w_{R}\right| d x\right.
$$


and

$$
(m / \varepsilon) \int_{\Delta i}\left(\Phi^{\prime}\left(w_{R} / m\right) \varepsilon\left|\nabla w_{R}\right| d x \leq(m / \varepsilon) \int_{\Delta i} \Psi\left(\Phi^{\prime}\left(w_{R}\right) / m\right)+\Phi\left(\varepsilon\left|\nabla w_{R}\right|\right) d x\right.
$$

then

$$
\int_{\Delta i} \Phi\left(w_{R}\right)\left|\nabla w_{R}\right| d x \leq(m / \varepsilon) \int_{\Delta i} \Psi\left(\Phi^{\prime}\left(w_{R}\right) / m\right)+\Phi\left(\varepsilon\left|\nabla w_{R}\right|\right) d x
$$

Since

$$
\Psi\left(\Phi^{\prime}\left(w_{R}\right) / m\right) \leq \Psi\left(w_{R} \Phi^{\prime}\left(w_{R}\right) /\left(m w_{R}\right) \leq \Psi\left(\Phi\left(w_{R}\right) / w_{R}\right)\right.
$$

from the inequality

$$
\Psi(\Phi(t) / t))<\Phi(t)
$$

(see inequality (6), page 230 of [1]) we have

$$
\int_{\Delta i} \Phi\left(w_{R}\right)\left|\nabla w_{R}\right| d x \leq(m / \varepsilon) \int_{\Delta i} \Phi\left(w_{R}\right)+\Phi\left(\varepsilon\left|\nabla w_{R}\right|\right) d x
$$

then

$$
\Phi\left(k_{i}-k_{i-1}\right)\left|B\left(k_{i}, v_{R}, 1\right)\right| \leq C_{S N}\left|B\left(k_{i}, v_{R}, 1\right)\right|^{1 / N}(m / \varepsilon) \int_{\Delta i} \Phi\left(w_{R}\right)+\Phi\left(\varepsilon\left|\nabla w_{R}\right|\right) d x
$$

Moreover, since $\Phi$ globally satisfies the $\Delta^{\prime}$-condition in $[0,+\infty)$, it follows

$$
\Phi\left(k_{i}-k_{i-1}\right)\left|B\left(k_{i}, v_{R}, 1\right)\right| \leq C_{S N}\left|B\left(k_{i}, v_{R}, 1\right)\right|^{1 / N}\left[(m / \varepsilon) \Phi\left(k_{i}-k_{i-1}\right)\left|\Delta_{i}\right|+\left(m c_{1} c_{G} \varpi(\varepsilon)\right) \int_{\Delta i} \Phi\left(\left|\nabla w_{R}\right|\right) d x\right]
$$

since

$$
\int_{\Delta i} \Phi\left(\left|\nabla w_{R}\right|\right) d x=\int_{\Delta i} \Phi\left(\left|\nabla v_{R}\right|\right) d x
$$

using Caccioppoli's inequality (2.2) we have

$$
\left.\left|B\left(k_{i}, v_{R}, 1\right)\right|\right|^{1-1 / N} \leq C_{S N}(m / \varepsilon)\left|\Delta_{i \mid}+C_{S N} m c_{2} \quad \varpi(\varepsilon)\right| Q_{2} \mid .
$$

Summing the last inequality on i from 0 to $v$ we have

$$
(1+v)\left|B\left(k_{i}, v_{R}, 1\right)\right|^{1-1 / N} \leq C_{S N}(m / \varepsilon)\left|Q_{1}\right|+C_{S N} m c_{2} \quad \varpi(\varepsilon)\left|Q_{2}\right|(1+v)
$$

and

$$
\left|B\left(k_{i}, v_{R}, 1\right)\right|^{1-1 / N} \leq C_{S N}(m /(\varepsilon(1+v)))\left|Q_{1}\right|+C_{S N} m c_{2} \quad \varpi(\varepsilon)\left|Q_{2}\right| .
$$

Fix $\varepsilon=\left(1 /(1+v)^{1 / 2}\right)$, then

$$
\left|B\left(k_{i}, v_{R}, 1\right)\right|^{1-1 / N} \leq C_{S N}\left(m /(1+v)^{1 / 2}\right)\left|Q_{1}\right|+C_{S N} m c_{2} \varpi\left(1 /(1+v)^{1 / 2}\right)\left|Q_{1}\right|
$$

and

$$
\left|B\left(k_{i}, v_{R}, 1\right)\right|^{1-1 / N} \leq C_{S N} m\left(1 /(1+v)^{1 / 2}+c_{2} \varpi\left(1 /(1+v)^{1 / 2}\right)\right)\left|Q_{1}\right|^{1-1 / N}
$$

From (2.9) we have

$$
\left|B\left(k_{i}, v_{R}, 1\right)\right| \leq\left(C_{S N} m\right)^{N /(N-1)}\left(1 /(1+v)^{1 / 2}+c_{2} \varpi\left(1 /(1+v)^{1 / 2}\right)^{N /(N-1)}\left|Q_{1}\right| .\right.
$$

Since $\varpi(s) \downarrow 0$ for $s \downarrow 0$ then we can choose $v$ such that

$$
\left(C_{S N} m\right)^{N /(N-1)}\left(1 /(1+v)^{1 / 2}+c_{2} \varpi\left(1 /(1+v)^{1 / 2}\right)^{N /(N-1)} \leq(1 / 2)\left(\delta_{0}\right)^{(N-1) N}\right.
$$

where $\delta_{0}$ is the constant in Lemma 7, then there exists $\lambda\left(\delta_{0}\right)$ such that

$$
\inf _{Q R / 2}\{u\} \geq \lambda(\delta) \theta \text {. }
$$


Let now $\mathrm{T}>(1 / 2)$ and assume $|\mathrm{B}(\theta, \mathrm{u}, \mathrm{R})| \leq \delta\left|\mathrm{Q}_{\mathrm{R}}\right|$ and u positive in $\mathrm{Q}_{2 \mathrm{R}}$. Since

$$
|A(\theta, u, 2 T R)||\geq(1-\gamma)| Q_{R}\left|=\left((1-\delta) /(2 T)^{\mathrm{n}}\right)\right|\left|Q_{2 T R}\right|
$$

we have

$$
|B(\theta, u, 2 T R)| \leq\left(1-(1-\delta) /(2 T)^{\mathrm{n}}\right)\left|Q_{2 T R}\right|
$$

then there exists a constant depending on $\delta$ and T such that (2.4) holds.

Using the technique introduces in [11] we get the following lemma.

Lemma 9: Let $\mathrm{u} \in \mathrm{DG}_{\Phi}{ }^{-}$with $\mathrm{k}_{0}=0$ and let $\mathrm{u}$ be positive in $\mathrm{Q}_{2}$. Let $\delta \in(0,1)$ and $\mathrm{t}>0$. If

$$
\left|\left\{x \in Q_{1}: u(x)>t\right\}\right| \geq 2^{-s}\left|Q_{1}\right|
$$

then

$$
\inf _{Q 1 / 2}\{u\}>c^{s} t
$$

where $\mathrm{c}=\mathrm{c}(\delta)$ being as in Lemma 8 with $\delta=2^{-\mathrm{N}-1}$.

Proof: For $\mathrm{s}=0$ the claim is true by Lemma 8 . Now we use the inductive process. We assume the claim true for some $\mathrm{s}$ and we prove it for $\mathrm{s}+1$. Let us define $\mathrm{A}_{\mathrm{i}}=\left\{\mathrm{x} \in \mathrm{Q}_{1}: \mathrm{u}(\mathrm{x})>\mathrm{c}^{\mathrm{i}} \mathrm{t}\right\}$; by hipothesis, if $\mathrm{A}_{0}=\left\{\mathrm{x} \in \mathrm{Q}_{1}: \mathrm{u}(\mathrm{x})>\right.$ t\} then

$$
\left|A_{0}\right|>\left(1 / 2^{s+1}\right)\left|Q_{1}\right|
$$

We have two alternative.

1) We assume $\left|A_{0}\right|>2^{-s}\left|Q_{1}\right|$, the by inductive hypothesis

$$
\inf _{Q / 2}\{u\}>c^{s} t>c^{s+1} t
$$

2) Otherwise $2^{-\mathrm{s}-1}\left|\mathrm{Q}_{1}\right|<\left|\mathrm{A}_{0}\right|<2^{-\mathrm{s}}\left|\mathrm{Q}_{1}\right|$. Let us assume $\mathrm{g}=\chi\left\{\mathrm{A}_{0}\right\}$ and apply the Calderon-Zygmund argument to $g$ in $Q_{1}$ with parameter $(1 / 2)$ then we find a sequence of dyadic cubes $\left\{\mathrm{Q}_{\mathrm{j}}\right\}$ such that

$$
\begin{gathered}
(1 / 2)<\left(1 /\left|Q_{j}\right|\right) \int Q_{j} g d x<2^{N-1} ; \\
g<(1 / 2) \text { in } Q_{1} \backslash \bigcup_{j} Q_{j} ;
\end{gathered}
$$

if $\mathrm{Q}_{\mathrm{j}}$ is one of the $2^{\mathrm{N}}$ subcubes of $\mathrm{P}_{\mathrm{i}}$ arising during the Calderon-Zygmund process, then

$$
\left(1 /\left|P_{i}\right|\right) \int_{P i} g d x \leq(1 / 2) \text {. }
$$

From (2) and (3) we get

$$
\left|A_{0}\right|=\left|A_{0} \cap \bigcup_{i} P_{i}\right|=\sum_{i}\left|A_{0} \cap P_{i}\right| \leq(1 / 2) \sum_{i}\left|P_{i}\right|
$$

moreover

$$
\left|A_{0} \cap P_{i}\right| \geq\left|A_{0} \cap Q_{\lambda}\right| \geq(1 / 2)\left|Q_{j} \geq\left(1 / 2^{N+1}\right)\right| P_{i} \mid .
$$

We apply Lemma 8 and we obtain

$$
\inf _{P i}\{u\} \geq c t
$$

Let us consider

$$
A_{1}=\left\{x \in Q_{1}: u(x)>c t\right\}
$$

then $\mathrm{P}_{\mathrm{i}} \subset \mathrm{A}_{1}$ and

$$
\left|Q_{1}\right| 2^{-s-1}<\left|A_{0}\right|<(1 / 2)\left|A_{1}\right|
$$

by inductive hypotesis

$$
\inf _{Q 1 / 2}\{u\}>c^{s+1} t
$$




\subsection{Proof of the Weak Harnack Inequality}

Now we can proof the inequality (1.19) using the technique introduced by Di Benedetto-Trudinger in [6].

Proof (Proof of Theorem 5); Given any $t>0$ choose an integer s such that

$$
\lambda_{t}=\left|\left\{x \in Q_{R}: u(x)>t\right\}\right| \geq 2^{-s}\left|Q_{R}\right|
$$

i.e.

$$
s \geq \ln \left(\lambda_{t} / Q_{R} \mid\right) / \ln (1 / 2) ;
$$

then by Lemma 9 we get

$$
\text { ess }-\inf f_{Q R / 2}\{u\}>c^{s} t
$$

therefore

$$
u(x) \geq t\left(\lambda_{t} /\left|Q_{R}\right|\right)^{\ln (c) / \ln (1 / 2)} .
$$

Let us define

$$
\xi=e s s-i n f_{Q R / 2}\{u\}
$$

then

$$
\lambda_{t} \leq\left(\xi^{\alpha} / t^{\alpha}\right)\left|Q_{R}\right|
$$

where $\alpha=\ln \left((1 / 2) / \ln (\mathrm{c})\right.$. Since $\Phi^{\prime}(\mathrm{t}) \mathrm{t} \leq \mathrm{m} \Phi(\mathrm{t})$ for $\mathrm{p}>\max \{1,(\mathrm{~m} / \alpha)\}$ we have

$$
\int_{Q R} \Phi^{1 / p}(u) d x=(1 / p) \int_{[0,+\infty]} \Phi^{1 / p-1}(t) \Phi(t) \lambda_{t} d t \leq(1 / p) \Phi^{1 / p}(\xi)\left|Q_{R}\right|+(m / p)\left|Q_{R}\right| \xi^{\alpha} \int_{[\xi,+\infty]} \Phi^{1 / p}(t) / t^{\alpha+1} d t
$$

Integrating by parts, we have

$$
\int_{[\xi,+\infty]} \Phi^{1 / p}(t) / t^{\alpha+1} d t \leq(1 /(\alpha[1-(m /(p \alpha))])) \Phi^{1 / p}(\xi) \xi^{-\alpha}
$$

hence

$$
\left(1 /\left|Q_{R}\right|\right) \int_{Q R} \Phi^{1 / p}(u) d x \leq c \Phi^{1 / p}\left(e s s-i n f_{Q R / 2}\{u\}\right) .
$$

\section{References}

[1] Adams, R. (1975) Sobolev Spaces. Accademic Press, New York.

[2] Ambrosio, L. Lecture Notes on Partial Differential Equations.

[3] Astarita, G. and Marrucci, G. (1974) Principles of Non-Newtonian Fluid Mechanics. McGraw-Hill, London.

[4] Bhattacharaya, T. and Leonetti, F. (1993) $\mathrm{W}^{\wedge}\{2,2\}$ Regularity for Weak Solutions of Elleptic Systems with Nonstandard Growth. J. Math. Anal. Appl., 176, 224-234. http://dx.doi.org/10.1006/jmaa.1993.1210

[5] De Giorgi, E. (1957) Sulla differenziabilità e l'analicità delle estremali degli integrali multipli. Mem.Accad. Sci Torino, cl. Sci. Fis. Mat. Nat., 3, 25-43.

[6] Di Benedetto, E. and Trudinger, N. (1984) Harnack Inequalities for Quasi-Minima of Variational Integrals. Ann. Inst. H. Poincaré (analyse non lineaire), 1, 295-308.

[7] Fuchs, M. and Seregin, G. (1998) A Regularity Theory for Variational Intgrals with LlnL-Growth. Calc. Var., 6, 171-187. http://dx.doi.org/10.1007/s005260050088

[8] Fuchs, M. and Mingione, G. (2000) Full $C^{\wedge}\{1, \alpha\}$-Regularity for Free and Constrained Local Minimizers of Elliptic Variational Integrals with Nearly Linear Growth. Manuscripta Mathematica, 102, 227-250. http://dx.doi.org/10.1007/s002291020227

[9] Giaquinta, M. and Giusti, E. (1982) On the Regularity of Minima of Variational Integrals. Acta Mathematica, 148, 31-46. http://dx.doi.org/10.1007/BF02392725

[10] Giaquinta, M. and Giusti, E. (1984) Quasi-Minima. Ann. Inst. H. Poincarè (Analyse non lineaire), 1, 79-107.

[11] Giaquinta, M. and Martinazzi, L. (2005) An Introduction to the Regularity Theory for Elliptic Systems, Harmonic Maps and Minimal Graphs. S.N.S. press, Pisa. 
[12] Giusti, E. (1994) Metodi diretti nel Calcolo delle Variazioni. U. M. I., Bologna.

[13] Gosez, J.P. (1974) Non Linear Elliptic Problems for Equations with Rapidly (or Slowly) Increasing Coefficents. Transactions of the American Mathematical Society, 190, 163-205. http://dx.doi.org/10.1090/S0002-9947-1974-0342854-2

[14] Granucci, T. (2014) An Alternative Proof of the Hölder Continuity of Quasi-Minima of Scalar Integral Functionals with General Growths. Afr. Mat., 25,197-212. http://dx.doi.org/10.1007/s13370-012-0109-3

[15] Granucci, T. An Alternative Proof of the Hölder Continuity of Quasi-Minima of Scalar Integral Functionals with General Growths. Part II. Submit to Afrika Matematika.

[16] Granucci, T. Observations on $\mathrm{L}^{\Phi}-\mathrm{L}^{\infty}$ Estimations and Applications to Regularity. Submit to Indian Journal of Pure and Applied Mathematics.

[17] Lieberman, G.M. (1991) The Natural Generalization of the Natural Conditions of Ladyzhenskaya and Ural'tseva for Elliptic Equations. Communications in Partial Differential Equations, 16, 331-361. http://dx.doi.org/10.1080/03605309108820761

[18] Klimov, V.S. (2000) Embedding Theorems and Continuity of Generalized Solutions of Quasilinear Elliptic Equations. Differential Equation, 36, 870-877. http://dx.doi.org/10.1007/BF02754410

[19] Krasnosel'skij, M. A. and Rutickii, Ya.B. (1961) Convex Function and Orlicz Spaces. Noordhoff, Groningen.

[20] Marcellini, P. (1993) Regularity for Elliptic Equations with General Growth Conditions. Journal of Differential Equations, 105, 296-333. http://dx.doi.org/10.1006/jdeq.1993.1091

[21] Maercellini, P. (1996) Regularity for Some Scalar Variational Problems under General Growth. Journal of Optimization Theory and Applications, 1, 161-181. http://dx.doi.org/10.1007/BF02192251

[22] Marcellini, P. (1996) Everywhere Regularity for a Class of Elliptic Systems without Growth Conditions. Annali della Scuola Normale Superiore di Pisa, 23, 1-25.

[23] Mascolo, E. and Papi, G. (1994) Local Bounddeness of Minimizers of Integrals of the Calculus of Variations. Annali di Matematica Pura ed Applicata, 167, 323-339. http://dx.doi.org/10.1007/BF01760338

[24] Mascolo, E. and Papi, G. (1996) Harnack Inequality for Minimizer of Integral Functionals with General Growth Conditions. Nonlinear Differential Equations and Applications, 3, 231-244. http://dx.doi.org/10.1007/BF01195916

[25] Mingione, G. and Siepe, F. (1999) Full $C^{\wedge}\{1, \alpha\}$ Regularity for Minimizers of Integral Functionals with L $\operatorname{logL}$ Growth. Zeitschrift für Analysis und ihre Anwendungen, 18, 1083-1100. http://dx.doi.org/10.4171/ZAA/929

[26] Moscariello, G. and Nania, L. (1991) Hölder Continuity of Minimizers of Functionals with Nonstandard Growth Conditions. Ricerche di Matematica, 15, 259-273.

[27] Nash, J. (1958) Continuity of Solutions of Parabolic and Elliptic Differential Equations. American Journal of Mathematics, 80, 931-953. http://dx.doi.org/10.2307/2372841

[28] Rao, M.M. and Ren, Z.D. (1991) Theory of Orlicz Spaces. Marcel Dekker, New York.

[29] Talenti, G. (1990) Bounddeness of Minimizers. Hokkaido Mathematical Journal, 19, 259-279. http://dx.doi.org/10.14492/hokmj/1381517360 